\title{
Variations
}

Variations

Revue internationale de théorie critique

$13 / 14 \mid 2010$

Le choix du petit

\section{La revanche des publics à l'attaque des industries culturelles}

Julien Bordier

\section{(2) OpenEdition}

Journals

Édition électronique

URL : http://journals.openedition.org/variations/177

DOI : 10.4000/variations. 177

ISSN : 1968-3960

Éditeur

Les amis de Variations

Édition imprimée

Date de publication : 31 mars 2010

\section{Référence électronique}

Julien Bordier, «La revanche des publics à l'attaque des industries culturelles », Variations [En ligne],

13/14 | 2010, mis en ligne le 01 février 2012, consulté le 20 avril 2019. URL : http://

journals.openedition.org/variations/177 ; DOI : 10.4000/variations.177

Ce document a été généré automatiquement le 20 avril 2019.

Les ami•e•s de Variations 


\title{
La revanche des publics à l'attaque des industries culturelles
}

\author{
Julien Bordier
}

\section{NOTE DE L'ÉDITEUR}

Première publication sur www.theoriecritique.com, « Le choix du petit », Printemps 2010, pp. 50-65

1 Prêter attention à la pratique du téléchargement illégal de fichiers culturels sur Internet est une occasion d'adopter une approche renouvelée des rapports entre créateurs, publics, politiques publiques et industries culturelles. On peut en effet se demander si le téléchargement n'est pas la pratique la plus subversive qui ait émergée dans le domaine de la culture ces dernières années. D'autre part, on ne peut nier la force de polarisation démocratique qu'a mis en évidence le débat sur la création de la Haute Autorité pour la Diffusion des CEuvres et la Protection des droits sur Internet. Rarement une question culturelle a si largement débordé les frontières admises de la mise en discussion publique : quittant la sphère habituelle du débat d'experts culturels, pour donner lieu à une effervescence rebelle inattendue. Il suffit de saisir le terme «HADOPI » dans un moteur de recherche pour le constater.

2 Pour comprendre la dimension inédite introduite par le téléchargement illégal, il est d'abord nécessaire de redéfinir en quoi consiste cette pratique. Ce passage par une définition de base est nécessaire pour mettre de côté des accusations fantaisistes selon lesquelles, par exemple, le téléchargement financerait d'importantes organisations criminelles et terroristes ${ }^{1}$. Cette mise au point permettra également de dépasser la question du vol, posée par les pouvoirs publics, pour aller au contraire vers celui du partage.

On s'interrogera ensuite sur les relations qu'il est possible d'établir entre les politiques culturelles mises en place par l'Etat et le téléchargement illégal. Au moment où le 
ministère de la Culture fêtait son cinquantenaire, il se lançait dans une croisade répressive de grande envergure contre la pratique du téléchargement. Inscrit dans une perspective historique, on verra que ce hasard du calendrier produit du sens.

4 Enfin, de manière plus générale, je souhaite proposer une lecture de ce phénomène comme une invention et une intervention politique inédite, et pas seulement comme la découverte d'un nouvel outil, contrariant le monde tel qu'il va, en particulier l'hégémonie des industries culturelles et le peu de place laissée à la subjectivité et l'autodétermination des publics dans les problématiques artistiques. Pour le dire avec d'autres mots, je soutiendrai l'idée que la pratique du téléchargement anticipe et dépasse des formes de propriété et d'échange qui montrent leurs limites dans la crise du capitalisme. Cet enjeu est sûrement d'autant plus particulier qu'il émerge dans le domaine artistique, où le principe d'émancipation est presque toujours en jeu, et du côté où on ne l'attend pas, c'est-à-dire de celui des publics, en tout cas de ceux qui sont identifiés comme tel.

\section{Lîle aux trésors des pirates généreux}

Définir clairement - et donc techniquement - ce qu'est le téléchargement de contenus culturels sur Internet révèle d'ores et déjà des idées importantes : cela permet à ceux qui ont une idée très vague du phénomène de comprendre la simplicité du procédé, cette compréhension permet de "démonter » les arguments du discours anti-téléchargement et enfin d'insister sur des valeurs qui sont au cœur de la pratique, à savoir le partage et la mise en commun.

Il faut préciser pour commencer que tout téléchargement n'est pas illégal. Il sera donc question ici de la pratique que la création de l'HADOPI cherche à sanctionner, c'est-à-dire, l'échange de fichiers culturels - films et musiques notamment, mais aussi jeux ou autres logiciels payants - soumis au code de la propriété intellectuelle.

7 Il existe différents procédés de partages illégaux de fichiers culturels sur Internet, comme le Peer2Peer ou les systèmes BitTorrent. À partir d'une interface informatique - logiciel et/ou page internet - on cherche un film, de la musique ou un jeu, convertis en fichiers numériques. Une fois le fichier trouvé, un protocole d'échange permet d'aller le télécharger (download) sur le disque dur d'un autre utilisateur. Plus un fichier identique est présent chez de nombreux utilisateurs, plus il sera aisé de l'acquérir. Au moment où le fichier est acquis par l'utilisateur, il se met en principe lui-même à le fournir à d'autres utilisateurs (upload). Partage et mise en commun sont donc au centre de la pratique. Reste à comprendre comment un premier fichier est devenu disponible et accessible à d'autres utilisateurs. Il suffit d'inventivité et d'imagination pour le comprendre. Tous les scénarii sont possibles : j'emprunte, je loue ou j'achète un DVD ou un CD et je le convertis dans un format partageable; je suis journaliste et je reçois des DVD ou CD promotionnels et je les convertis puis partage ; je suis salarié d'une maison de production et j'ai accès à des exemplaires promotionnels; je suis persuadé de la valeur du partage de films et je me rends au cinéma avec une caméra de poche pour faire une copie de la projection en vue de la partager, etc. Pour être bien clair, convertir signifie dématérialiser dans le cas du DVD ou du CD, transformer en données numériques sur mon propre disque dur. Dans l'exemple de celle qui re-filme une projection cinématographique, on pourrait dire que le film est re-matérialisé sur un support numérique en vue de la partager. Il y a là un étonnant de mouvement de dématérialisation (des produits culturels), rematérialisation (par les pirates) puis re-dématérialisation (dans le partage). Dans tous les cas, l'idée est de 
disposer de données numériques légères qui pourront aisément se balader sur la toile, dans des formats lisibles par le plus grand nombre d'utilisateurs - tels que les encodages mp3 pour la musique ou DivX pour la vidéo.

À proprement parler, ce sont ceux et celles qui fournissent ce fichier originel qui sont les pirates. Les autres, en le téléchargeant, ne procèdent qu'au partage du fichier, c'est à ce moment-là du processus qu'ils deviennent eux-mêmes pirates selon les lois de protection de la propriété intellectuelle. On devrait donc parler de piratage/partage.

Plus un fichier est présent chez de nombreux utilisateurs, plus il est disponible et donc téléchargeable. En d'autres termes, plus des utilisateurs accumuleront des fichiers partagés, plus le fond partageable sera élargi et la disponibilité des fichiers facilitée. Pour reprendre les termes employés par les utilisateurs du protocole BitTorrent par exemple, un leecher (une sangsue) qui cherche à acquérir un fichier, deviendra Seed (une semence) une fois le téléchargement terminé.

Se développe ainsi de manière illégale une colossale bibliothèque de fichiers culturels qui permet un accès gratuit à un nombre considérable d'œuvres - susceptibles d'être fixée dans un format numérique facilement partageable. On peut voir ce mouvement comme une occasion d'accès du plus grand nombre à la production culturelle. Il faut de plus souligner les efforts développés par certains - qu'ils créent ou développent les interfaces de partage, qu'ils créent les fichiers, qu'ils les contrôlent, qu'ils commentent leur qualité, qu'ils stockent des données - pour alimenter ce fond. Animer la communauté des pirates/ partageux ${ }^{2}$ prend du temps et ne rapporte, dans la plus grande majorité des cas, rien d'autre que la satisfaction de créer cet accès libre à des biens culturels. La doxa économique y laisse des plumes : le bénéfice en argent est mis de côté, la question du don, et de ses corollaires subversifs, est fortement activée.

11 Cette communauté s'invente, grâce à la mobilisation des publics. Il est relativement inédit de voir apparaître un mouvement d'accès à la culture, sans impulsion des créateurs ou des pouvoirs publics. De plus il faut remarquer que ce mouvement se construit sans principe politique fondateur - si ce n'est le partage mais qui n'est pas systématiquement mobilisé de manière militante - et sans établir de barrière entre cultures légitimes et illégitimes. Et en passant outre les codes de la propriété intellectuelle.

12 Ces réseaux d'échanges mettent en pratique une forme de potlatch numérique, mais qui n'implique ni destruction, ni désappropriation de la part de ceux qui donnent (upload) et reçoivent (download). Une éthique pirate se crée, consciente du besoin de reconnaissance des créateurs, tout en étant avide de partage, d'échange, de mise en commun par et pour les publics.

13 Si on considère ce libre accès comme un manque à gagner pour les créateurs et/ou producteurs des biens culturels partagés, il faut remarquer l'originalité de ce phénomène où un processus d'accumulation de biens culturels contrarie celui de l'accumulation de capital. De fait, les fichiers contenant des œuvres issues des grosses industries culturelles - on pense ici en particulier au cinéma de type blockbusters, à la musique produite par des majors - sont les plus accessibles. Ce sont ces œuvres qui par le jeu de la diffusion médiatique ont besoin et mettent en place des opérations promotionnelles de grandes envergures. Elles donnent alors l'occasion malgré elles d'être largement piratées. Par exemple, les films les plus attendus et les plus secrets, se retrouvent sur la toile avant même d'être projetés sur les écrans de cinéma. L'attente, voire l'impatience, qui cherche à être créée chez le spectateur/consommateur par tous les moyens publicitaires possibles 
est donc totalement subvertie. En consultant le site Torrentfreak ${ }^{3}$ et ses classements réguliers des films les plus partagés, on constate clairement que ce sont exclusivement les très grosses productions hollywoodiennes qui sont les plus accessibles, les plus disponibles et donc les plus partagées. Ici encore les arguments cherchant à légitimer une répression du téléchargement se montrent donc caduques : le piratage/partage ne semble pas nuire aux petits artistes et à la production indépendante. En reprenant le cas du cinéma, et dans le contexte français, la dernière enquête sur les pratiques culturelles commandée par le ministère de la Culture montre au contraire que la fréquentation occasionnelle des salles obscures augmente ${ }^{4}$.

Enfin, recentrer la question du téléchargement sur la notion de partage contredit de manière radicale la manière dont ses détracteurs s'appliquent à présenter ceux qui téléchargent comme de simples voleurs. L'enjeu-de-mot sémantique autour des termes «piratage» et "partage» est de taille. Difficile d'imaginer les adversaires de ces pratiques dénoncer le "partage» dans des législations qui «criminaliseraient le partage ». La contradiction entre le discours publicitaire du " connecting people», de "l'échange ", du «être ensemble » vanté par l'industrie du spectacle et les médias de masse, et la mise en pratique effective de cet ensemble connecté, devient flagrante. Les mots ont une importance. Ceux et celles qui téléchargent deviennent des voleurs, comme le montrent les spots anti-pirates présents en introduction de la plupart des films en location en vidéos-clubs. Le message est clair: vous ne voleriez pas une voiture, vous n'arracheriez pas un sac à main, vous ne cambrioleriez pas un domicile, alors vous ne devriez pas télécharger, car télécharger c'est voler ${ }^{5}$. Les adeptes du piratage/partage semblent clamer un discours diamétralement opposé, ravivant des idées que la normalisation économique croyait avoir fait oublier : la propriété c'est le vol.

Il est aisé de comprendre pourquoi il est nécessaire d'assimiler le téléchargement à du vol et de ne jamais évoquer le principe de partage qui lui sert de fondement et d'éthique. Il s'agit de culpabiliser, d'insister sur la dimension immorale de la pratique, en éluder les vertus partageuses pour mieux la criminaliser. Au-delà de la criminalisation, ce qu'il faut déceler c'est bien sûr un projet contrariant pour la logique économique : d'autres formes d'échanges sont possibles et pratiquées. Criminaliser les pratiques revient à rendre illégal des formes de dépassement de l'échange capitaliste.

\section{De la démocratisation à la démocratie}

En 2009, le ministère de la Culture célébrait son cinquantenaire. Il se lançait aussi dans le projet de loi « favorisant la diffusion et la protection de la création sur Internet. » Celui-ci s'inspirait du rapport de 2007 Le développement et la protection des cuvres culturelles sur les nouveaux réseaux ${ }^{6}$, commandé par la ministre de la Culture Christine Albanel à Denis Olivennes. Cet ancien haut fonctionnaire connaît bien le monde des industries de la culture. Il fut tour à tour président directeur général de NC Numéricable, président puis directeur général de Canal + France, directeur général distribution dans le groupe PPR et ainsi nommé directeur général du groupe FNAC. Il est depuis 2008 directeur général délégué du Nouvel Observateur. Souvent cité dans la presse numérique pour avoir qualifié Internet de "tout-à-l'égout de la démocratie ", cet inspirateur de la loi dite Hadopi symbolise bien les liens étroits entre le ministère de la Culture et les industries culturelles. Le ton du rapport illustre bien quant à lui l'orientation que suivra la loi. $\mathrm{Ce}$ texte est principalement consacré aux mesures répressives, quelles soient techniques ou 
judiciaire, pour limiter le téléchargement de fichiers culturels soumis aux droits d'auteurs. Les acteurs du mouvement n'y ont nulle part leur mot à dire. Comment est-on passé du logos de la démocratisation de la Culture à la répression de son partage?

Le principe fondateur du Ministère de la Culture depuis sa création est «de rendre accessibles au plus grand nombre les œuvres capitales de l'humanité», ce qui s'est ensuite traduit dans le concept de démocratisation culturelle. Mais si ce terme fait aujourd'hui consensus dans le lexique politique, au même titre que exception culturelle, et revendique une certaine fierté nationale, il est utile d'en rappeler les origines.

Les missions du ministère des Affaires Culturelles de Malraux de 1959 sont souvent présentées comme novatrices. C'est en effet avec lui pour la première fois qu'il existe un véritable ministère d'État chargé de mettre en place des politiques culturelles. Mais contrairement à une certaine mythologie républicaine, il représente davantage une pérennisation qu'un véritable tournant institutionnel. Les missions du ministère malruxien sont héritées d'une longue tradition. Formulées par la philosophie des Lumières, ces idées trouvent leurs premières applications institutionnelles à partir de la Révolution française. On peut même penser que les idéaux culturels révolutionnaires ne font que « républicaniser » le projet monarchique de muséum royal, accessible au public, qui voit le jour à la fin du règne de Louis XVI. Bien sûr, la relecture révolutionnaire fait de la République elle-même la gardienne des arts au service des citoyens. Dès 1790, l'Assemblée constituante demande par décret de veiller à la conservation des monuments et biens culturels nationaux et une Commission Temporaire des Arts est formée en 17937, année qui voit aussi l'ouverture du musée du Louvre.

Cette tradition d'intervention de l'Etat dans le domaine culturel n'a pas déclinée sous les différentes Restaurations. Puis, elle s'est particulièrement institutionnalisée dans le système des Beaux-Arts de la IIIe République. Si quelques épisodes historiques peuvent le laisser penser, on ne peut pas affirmer que la finalité d'un tel interventionnisme fut la création d'une esthétique officielle. L'objectif a toujours plutôt été d'utiliser les biens culturels à des fins d'instruction publique. Mais la frontière séparant cette dernière de la propagande politique est poreuse. «Il n'est pas de témoignage de culture qui ne soit en même temps un témoignage de barbarie ${ }^{8}$ " écrit Benjamin pour évoquer le destin des biens culturels. La création du ministère en 1959, rompt principalement avec cette tradition en insistant sur la relation plus esthétique qu'éducative entre l'œuvre et le public. Cela implique toujours de définir où commencent et où s'arrêtent les expressions artistiques et les expériences esthétiques vécues dans la constellation que composent les publics et les créateurs. Il suffit d'avoir une approche assez ouverte de la question pour comprendre qu'on ne peut résoudre ce problème. Quelles sont donc les « œuvres capitales de l'humanité " que les pouvoirs publics doivent s'appliquer à rendre accessible? Comment le point de vue des publics et la diversité des expressions peuvent-ils être pris en compte?

Les années Lang sont une autre étape importante dans l'histoire du ministère. Elles sont marquées par l'élargissement de son champ d'action, par l'intérêt porté à des pratiques plus diversifiées, mais aussi par le développement important des industries culturelles et leur prise en compte dans les politiques publiques. L'expression de « démocratisation de la culture » fait alors consensus pour synthétiser les missions du ministère. Il s'inscrit dans le continuum historique des prérogatives culturelles de l'Etat en France, en se concentrant sur le problème de l'accessibilité. Mais la démocratisation implique un arbitrage préalable pour déterminer ce qui doit être soutenu et diffusé pour être 
démocratisé. La diversité des expressions et interprétations culturelles, leur caractère insaisissable et subjectif, sont mis de côté. «Démocratiser la culture » signifierait «la culture de certains pour tous", contrairement à la «démocratie culturelle» qui signifierait « la culture de tous, par tous et pour tous. »

À l'évidence, dans la mesure où elles relèvent du secteur privé, et qu'elles génèrent beaucoup de profit, l'Etat ne peut théoriquement qu'intervenir à la marge dans les conditions de création et diffusion de toute une partie des biens culturels. Les politiques culturelles ont généralement cherchées à minimiser les conséquences de la loi du marché sur l'accès aux biens culturels, notamment en assurant des missions déficitaires et en compensant le manque à gagner pour les entreprises privées.

Dans le cas du piratage/partage, le manque à gagner pour le secteur privé est incalculable. Non pas parce qu'il est faramineux, mais parce qu'on peut difficilement savoir comment l'échange de contenus culturels influence ou non la consommation directe des produits des industries culturelles. Le parti pris de la répression n'est donc pas fondé logiquement: difficile de savoir si le partage encourage ou enraye la consommation de biens culturels. Pour légitimer la répression du partage, il est question de protection des créateurs. C'est d'ailleurs ce que laisse entendre le titre de la loi. Or, en place de protection des créateurs c'est une protection de leurs ayants-droits qui est mise en place. La question se décline certes différemment en fonction des types des biens culturels (films, musiques, logiciels qui sont les premiers concernés), mais de manière générale les conditions actuelles de la diffusion à grande échelle de ces biens culturels concernent davantage les structures de production qui font travailler des créateurs sous contrat et/ou qui cherchent aujourd'hui assurer différemment leurs ressources. Ce sont ces grosses structures qui comptent voir les droits qu'ils détiennent être protégés.

Une telle protection - et une gestion rationalisée des profits potentiels pouvant être réalisés sur un bien culturel - ne peut passer que par un contrôle strict de l'accès aux biens culturels par l'intermédiaire du marché. Le piratage/partage contrarie ces possibilités de contrôle de l'accès. En fait, il les pulvérise. Il s'agit donc de l'empêcher d'exister. Au lieu de chercher à développer le potentiel de "démocratisation » qui est à l'œuvre dans cette pratique, il va falloir le réprimer. Le ministère de la Culture va s'en charger et ainsi soutenir les industries culturelles, qui sont en effet à ses yeux des acteurs essentiels des dynamiques culturelles. On assiste alors à ce glissement des missions officielles de l'institution, de la facilitation de l'accès à un contrôle exclusif.

Au-delà de l'enjeu économique, il me semble que le choix de la posture répressive est aussi largement idéologique. En effet, le ministère de la Culture n'est actuellement pas adepte du principe de gratuité comme la ministre Albanel l'a montré dans ses déclarations sur l'expérimentation de la gratuité partielle dans les musées nationaux par exemple. Le ministère s'accorde sur ce point avec l'auteur du rapport sur Le développement et la protection des ceuvres culturelles sur les nouveaux réseaux, également auteur d'un livre intitulé La gratuité c'est le vol'. Il est certes difficile d'attendre autre chose d'un représentant des industries culturelles.

Les politiques culturelles auraient pourtant beaucoup de leçons, autres que répressives, à tirer du développement de la pratique du piratage/partage. Je me limiterai ici à des remarques d'ordre culturel, mais on verra sans peine qu'elles relèvent du politique. Cette autre dimension sera développée dans la partie suivante, même si cette présentation laisse à penser qu'il faudrait dissocier le culturel du politique, ce qui pose problème. 
26 La "démocratisation de la culture ", telle que nous la comprenons dans sa formation historique relève d'une promesse : celle de l'accès du plus grand nombre, au plus grand nombre de biens culturels. La répression du piratage/partage est au contraire la restriction de ce principe. On peut donc analyser le phénomène comme un dépassement de la promesse formulée par l'Etat et la manifestation de la soif culturelle qui traverse les publics. Le phénomène étant de plus une émanation des publics eux-mêmes, sans impulsion institutionnelle et sans barrière mise entre les différentes formes de biens culturels, il me semble que le piratage/partage crée les conditions d'une démocratie culturelle. Celle-ci serait l'affirmation de la culture de tous, par tous et pour tous. L'abondance culturelle promise par la " démocratisation » se trouve créée par les publics eux-même. Je reviendrai plus loin sur l'importance du principe démocratique dans cette pratique.

À un niveau plus institutionnel, et pour finir de démonter les arguments antitéléchargement du ministère, il faut mentionner que la mise en téléchargement et le partage de biens culturels peuvent être vus comme une stimulation de l'offre culturelle. C'est ce que révèle un rapport néerlandais, Ups and downs. Economische en culturele gevolgen van file sharing voor muziek, film en games ${ }^{10}$ qui traite de la question du téléchargement avec beaucoup plus de subtilité que celui sur Le développement et la protection des oeuvres culturelles sur les nouveaux réseaux. S'il insiste sur les conséquences différentes du téléchargement dans les différents secteurs de l'industrie culturelle, il relativise fortement l'impact économique de la pratique en montrant que la production trouve les moyens de s'adapter à cette nouvelle situation, notamment en cherchant de nouvelles accroches qualitatives. Comme pour répondre aux nuances apportées par cette étude, le cabinet de consultants Tera vient de produire un rapport calculant les supposées retombées économiques du téléchargement : des centaines de milliards d'euro de manque à gagner et plus d'un million d'emplois détruits ${ }^{11}$. Cette catastrophe économique repose évidemment sur l'entière responsabilité des criminels qui téléchargent. La baisse tendancielle du taux de profit et la crise économique ne semblent avoir aucun impact sur les industries culturelles selon cette étude.

Pour conclure cette partie, j'aimerai illustrer les potentialités du partage de fichiers à partir d'un exemple, celui de l'album réunissant Sparklehorse, Danger Mouse et David Lynch sorti en 2009: Dark Night of the Soul. En raison d'un contentieux juridique entre Danger Mouse et la maison de disque EMI sur laquelle était signé l'artiste Sparklehorse, un des plus riches disques de l'année a failli ne pas voir le jour. C'est donc un disque vierge qu'a sorti le trio dans le commerce, agrémenté d'un livret de photos de David Lynch. Quant au contenu musical du disque, il a été placé en téléchargement libre sur internet. L'initiative largement relayée par les internautes, a rendu le disque accessible au plus grand nombre. Cette anecdote montre à mon avis, la nocivité des industries culturelles sur la création d'une part, et les potentialités inattendues du partage de fichiers d'autre part ${ }^{12}$.

\section{Le téléchargement contre le pouvoir}

Les enjeux culturels du piratage-partage esquissés, les arguments de ses détracteurs en partie démantelés, on peut maintenant s'intéresser aux potentialités politiques que recèlent la pratique du piratage/partage. Comme écrit plus haut, cette pratique est un contre-pouvoir face aux industries culturelles. J'aimerais maintenant en proposer une 
lecture en m'appuyant sur trois auteurs préoccupés par la question du dépassement du pouvoir et attentifs aux subjectivités politiques. L'enjeu est de montrer l'importance des angles de vue adoptés en sciences sociales pour nommer et déchiffrer l'inattendu, et d'autre part de tenter de rattacher des approches théoriques à des mises en pratiques. Les auteurs mobilisés seront Oskar Negt, James C. Scott et John Holloway. En quoi peuvent-ils nous aider à comprendre le piratage/partage ?

À travers le concept d'espace public oppositionnel ${ }^{13}$, Negt montre notamment que les institutions publiques et politiques classiques (des médias aux partis), admises et construites dans et par l'espace public bourgeois ne captent pas l'ensemble des pratiques et expressions politiques. Cela implique qu'une écoute sociologique particulière doit être mise en place pour cerner ce qui relève d'un espace public oppositionnel. Le piratage/ partage est une revendication forte du besoin d'accès aux biens culturels. Pour autant, cette revendication n'est pas exprimée dans les canaux classiques de la revendication politique : elle est assez peu reprise par l'opposition parlementaire, et ne donne pas lieu à des formes de mobilisation habituelles - en France en tout cas. De plus, la question de la légalité est centrale. Les pirates/partageux sont des criminels aux yeux de l'Etat et des lois internationales, ce qui ne semble pas les effrayer énormément. Le discours répressif est donc déconstruit par la pratique même. Les pirates/partageux refusent la loi du marché et celle de l'Etat et proposent d'aller plus loin dans l'accès aux biens culturels. Il est temps de dépasser les politiques culturelles semble être le mot d'ordre non formulé. En réalité, bien plus qu'être formulé, ce mot d'ordre est pratiqué. De plus, c'est la question du média qui peut polariser des subjectivités politiques qui est posée. Le piratage/partage offre deux issues inédites: il affirme de manière forte le besoin de médiation - c'est évidemment ici Internet et la mise en réseau qui compte - tout en rejetant les médiations classiques proposées par les partis et autres institutions de l'espace public bourgeois.

31 Au cœur de la théorisation proposée par Negt, il y a la subjectivité rebelle des acteurs. On ne peut négliger, comme déjà mentionné plus haut, l'importance du travail fourni par nombre de ceux et celles qui pratiquent le piratage/partage : il y a là un engagement actif qui ne dit pas son nom. Autre dimension essentielle, il est question de biens culturels. Ce qui se constitue à travers les réseaux de partage, c'est une communauté esthétique multiculturelle et aux facettes multiples, minimisant la place des experts et ouvrant la porte au plus grand nombre.

32 La domination n'est pas un processus parfait et contrairement à ce que les sciences sociales ont tendance à observer, elle ne se construit pas uniquement sur la servitude volontaire des dominés. Telle est la thèse de James $\mathrm{C}$. Scott développé à travers sa théorie du texte caché ${ }^{14}$. En s'appuyant sur des exemples de domination politique totale comme l'esclavage, l'auteur montre que toute relation de domination est inséparable de stratégies de résistance plus ou moins visibles pour la contrecarrer. Au-delà de ce constat, Scott montre même que la domination ne cesse de chercher à entrevoir le texte caché pour le détruire ou le désarmer en s'y adaptant. Je crois que l'ensemble des pratiques des internautes souhaitant avoir un large accès aux biens culturels relève d'un texte caché.

Aujourd'hui la plupart de ces pirates/partageux ne s'active pas à combattre la répression du téléchargement en suivant les normes dominantes. Au contraire, leur préoccupation est davantage de rendre la répression impossible. Ils adaptent donc leurs pratiques, soit en utilisant des vecteurs ne les faisant pas tomber sous le coup de la loi, soit en développant des techniques qui leur permettent d'être indétectable sur le réseau. Les stratégies de critique de la domination théorisée par Scott s'appuient en effet, comme 
l'expression « texte caché » le laisse entendre, sur une recherche des moyens de rendre la contestation invisible aux yeux des dominants pour éviter sanctions et représailles. L'analogie avec les techniques mises en place pour se rendre invisible sur le réseau pour pouvoir continuer à pirater/partager est parfaite.

Enfin, proposer une analyse en ces termes de la relation du public aux industries culturelles permet également de renouveler et d'adapter le discours existant jusqu'ici où l'idée principale des penseurs critiques reste la force d'aliénation des industries culturelles telles que pensées par Adorno et Horkheimer ${ }^{15}$, et/ou de l'industrie spectaculaire telle que pensée par les situationnistes. L'une comme l'autre présente le public, ou le spectateur, comme le dominé dans la relation de domination crée par la production de biens culturels. Restait à savoir quel était le texte caché qui se manifestait dans cette relation : le téléchargement apparaît comme en étant une partie.

On peut aller encore plus loin dans cette interprétation en mobilisant le concept d'antipouvoir proposé par John Holloway ${ }^{16}$. Tout comme Scott d'ailleurs, ce dernier montre que le pouvoir est toujours en retard sur ceux et celles qui le contestent. C'est ce qu'illustre l'utilisation de nouvelles techniques par les internautes pour contrecarrer la répression.

Le piratage/partage est-il un anti-pouvoir ? Il l'est assurément si on le considère comme un refus de la marchandisation des biens culturels et une volonté de mise en commun. Il contrarie le principe d'accumulation de capital par les industries culturelles et celui de la répression organisée par l'Etat. Or il ne cherche pas non plus à se constituer lui-même en pouvoir.

Une dimension importante de la réflexion d'Holloway repose sur l'attention portée à toutes formes de critique du monde tel qu'il va, même si elles ne rentrent pas dans les paradigmes politiques classiques. Toutes les prises de positions critiques et pratiques alternatives sont autant de failles dans la réification engendrée par le capitalisme. Ce sont ces multiples formes de la contestation, éphémères ou non, revendiquées comme telles ou non, qui constituent les anti-pouvoirs. Holloway déconstruit ainsi l'idée d'un pur sujet critique-révolutionnaire à l'identité ferme et rigide, l'identification étant présentée comme une forme de réification. Je pense que l'on peut affirmer que les pirates/ partageux ne sont pas de purs sujets critiques révolutionnaires. On ne peut pas décalquer sur leurs pratiques un schéma politique homogène. Le fond piraté/partagé en est luimême une bonne illustration: les œuvres les plus pointues et confidentielles y côtoient joyeusement les blockbusters des industries culturelles.

Enfin, on peut ajouter que le piratage/partage est un mouvement contre et au-delà ${ }^{17}$. Il se dresse contre la restriction de l'accès à certains biens culturels et va au-delà en proposant une alternative : une abondance et un libre accès à ces biens, basés sur le partage et la mise en commun. Le téléchargement est l'activation forte d'une utopie culturelle. Il est, et sera sûrement sans cesse, réprimé par les principes de réalité capitaliste. Ceux-ci ne peuvent l'interpréter que comme un manque à gagner. Rien ne sert d'attendre l'intervention de l'Etat pour protéger le partage. L'épisode Hadopi, malgré ses rebondissements institutionnels, vient bien confirmer que le ministère de la Culture s'aligne sur les positions des industries culturelles. Il faut par contre rester attentif à la manière dont les partisans du piratage/partage, ces petits et ces invisibles, vont continuer à ouvrir des brèches pour rendre le plus grand nombre d'œuvres accessibles au plus grand nombre. 


\section{NOTES}

1. Selon RAND Corporation, dont les études sont largement relayées dans les médias (par exemple dans l'article de C. Mulard, «La contrefaçon de DVD financerait le terrorisme » in Le Monde, 26 mars 2009), il y a des " preuves que les bénéfices du piratage financent le Hezbollah. » Voir www.rand.org/pubs/monographs/MG742/

2. Je reprends ici le terme qui définissait les partisans du partage des propriétés dans les mouvements de jacqueries ou pendant la Commune.

3. http://torrentfreak.com

4. Olivier Donnat, 2009. Les pratiques culturelles des français à l'ère numérique, La découverte/ Ministère de la Culture et de la Communication.

5. Preuve de la l'inventivité de la communauté partageuse, il faut remarquer que la campagne "Support artists not multinationals » a proposé un efficace détournement du spot en question. Voir http://www.iwouldntsteal.net/

6. Consultable à l'adresse: http://www.ladocumentationfrancaise.fr/rapportspublics/074000726/index.shtml

7. Bernard Deloche et Jean-Michel Deniaud, 1989. La culture des sans-culottes. Ed. de Paris/Presses du Languedoc.

8. Walter Benjamin, 2000 (1972). « Sur le concept d'histoire » in CEuvres III. Gallimard.

9. Denis Olivennes, 2007. La gratuité c'est le vol: quand le piratage tue la culture. Grasset.

10. Consultable en anglais à l'adresse http://www.ivir.nl/publicaties/vaneijk/ Ups_And_Downs_authorised_translation.pdf

11. Consultable en ligne à l'adresse http://www.teraconsultants.fr/assets/publications/PDF/ Executive-summary(français).pdf

12. Il faut rappeler que le contentieux opposant Danger Mouse à EMI est également lié à la question du téléchargement. Pour en savoir plus, j'invite le lecteur à écouter et se renseigner sur l'histoire du Grey Album, excitante rencontre entre le White Album des Beatles et le Black Album de Jay-Z.

13. Oscar Negt, 2007. L'espace public oppositionnel, Payot.

14. James C. Scott, 2008 (1992). La domination et les arts de la résistance, Editions Amsterdam.

15. Max Horkheimer, Theodor W. Adorno, 1974 (1944). La dialectique de la raison. Gallimard.

16. John Holloway, 2007 (2002). Changer le monde sans prendre le pouvoir. Lux.

17. John Holloway, 2006. «Un mouvement contre-et-au-delà.» In Variations, Parangon/ www.theoriecritique.com

\section{AUTEUR}

\section{JULIEN BORDIER}

Doctorant en sociologie, Paris 10 - Nanterre 